\title{
The Effects of Electron Beam Exposure Time on Transmission Electron Microscopy Imaging of Negatively Stained Biological Samples
}

\author{
Kyumin $\mathrm{Kim}^{\dagger}$, Jeong Min Chung ${ }^{1, \dagger}$, Sangmin Lee ${ }^{1, \dagger}$, Hyun Suk Jung ${ }^{1, \star}$ \\ Taejon Christian International School, Daejeon 34035, Korea \\ ${ }^{1}$ Department of Biochemistry, College of Natural Sciences, Kangwon National University, Chuncheon 24341, Korea
}

\author{
${ }^{\dagger}$ These authors contributed equally \\ to this work. \\ *Correspondence to: \\ Jung $\mathrm{HS}$ \\ Tel: +82-33-250-8513 \\ Fax: +82-33-259-5664 \\ E-mail: hsjung@kangwon.ac.kr
}

Received August 6, 2015

Revised September 15, 2015

Accepted September 17, 2015

\begin{abstract}
Negative staining electron microscopy facilitates the visualization of small bio-materials such as proteins; thus, many electron microscopists have used this conventional method to visualize the morphologies and structures of biological materials. To achieve sufficient contrast of the materials, a number of imaging parameters must be considered. Here, we examined the effects of one of the fundamental imaging parameters, electron beam exposure time, on electron densities generated using transmission electron microscopy. A single site of a negatively stained biological sample was illuminated with the electron beam for different times (1,2, or 4 seconds) and sets of micrographs were collected. Computational image processing demonstrated that longer exposure times provide better electron densities at the molecular level. This report describes technical procedures for testing parameters that allow enhanced evaluations of the densities of electron microscopy images.
\end{abstract}

Key Words: Bio-material imaging, Transmission electron microscopy, Single particle image processing, Electron beam exposure time

\section{INTRODUCTION}

A variety of bio-materials and proteins of different sizes and shapes have been studied using transmission electron microscopy (TEM). In this technique, high voltage electrons are used as an illumination source to produce a projected image containing details of the internal and external features of biological structures. The electron beam interacts with the sample under a vacuum as it passes through the entire thickness of the specimen, and contrast is generated by the scattering of electrons (Nagayama \& Danev, 2004; Massover, 2008; Murata et al., 2010; De Carlo \& Harris, 2011). Biological structures are easily distorted by dehydration caused by the vacuum of the microscope and radiation damage caused by the electron beam; hence, negative staining, a special specimen preparation technique, is commonly used to obtain sufficient contrast and preserve the native structure as much as possible (Brenner \& Horne, 1959; Monroe \& Brandt, 1970).

Using negative staining, the background is stained rather than the sample leaving it unstained; therefore, the image is formed from the difference between the non-stained area and the surrounding stained area. Good contrast is achieved when the stain surrounds the sample completely. Scattering of the electron beam by an object creates contrast; however, protein structures comprised of light atoms scatter the beam weakly and give poor contrast. Negative staining acts as a support to stabilize the biological structures, and enhances contrast to produce a well-defined electron density of the sample. Consequently, the electrons are scattered strongly by the stain and the TEM image is generated as a result of the contrast between differential scattering by the stain and the sample during the period of illumination.

This work was supported by research grants from Kangwon National University and a research supporting program for youth scientists (Kyumin Kim).

@ This is an open-access article distributed under the terms of the Creative Commons Attribution Non-Commercial License (http://creativecommons.org/licenses/by-nc/4.0) which permits unrestricted noncommercial use, distribution, and reproduction in any medium, provided the original work is properly cited.

Copyrights @ 2015 by Korean Society of Microscopy 


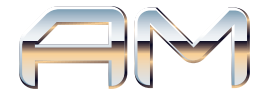

The advantages of negative staining over high resolution electron microscopy (EM) approaches, such as cryo-EM, are its convenience, simplicity, rapid applicability to biological objects ranging from individual proteins to large macromolecular assemblies, and ability to detect structural homologies (Anderson et al., 1989; Boekema et al., 2009; Zhang et al., 2013); hence, negative staining is used widely to detect the structural features of bio-materials and small proteins. To obtain the best contrast between stain and protein, the electron beam exposure time, which is related to the thickness of the support film, is an important parameter to consider.

Here, we investigated the effects of short and long electron beam exposure times on the TEM outcomes of negatively stained samples. We also describe technical procedures to identify the optimal exposure time that produces higher electron densities during TEM imaging. Single particle image averaging (Umeki et al., 2009; Jaitly et al., 2010) was used to process and compare the structural details obtained from a set of TEM images taken at three different times. Overall, the results suggest that longer exposures ( $2 \sim 4$ seconds) provide higher electron densities.

\section{MATERIALS AND METHODS}

\section{Negative Staining Electron Microscopy}

Negative staining was performed using purified thyroglobulin prepared from a protein stock (T9145; Sigma-Aldrich, USA). The stock protein was dissolved in phosphate-buffered saline (10 $\mathrm{mM}$ phosphate buffer, $2.7 \mathrm{mM} \mathrm{KCl}$, and $137 \mathrm{mM} \mathrm{NaCl}$; $\mathrm{pH}$ 7.4) and diluted with $20 \mathrm{mM}$ Tris- $\mathrm{HCl}$ buffer ( $\mathrm{pH}$ 7.5) to a final concentration of $100 \mathrm{nM}$. A $5 \mu \mathrm{L}$ aliquot of the diluted protein sample was applied to a carbon-coated grid that had been glow-discharged (Harrick Plasma, USA) for 3 minutes in air. The grid was immediately negatively stained using 1\% uranyl acetate (Jung et al., 2008; Jung et al., 2011; Elliott et al., 2012; Lee et al., 2015), and then examined using a Tecnai G2 Spirit Twin TEM system (FEI, USA) that was fitted with an anti-contaminator and operated at $120 \mathrm{kV}$. The analysis was performed using instrumentation at the Korea Basic Science Institute. Images were recorded using a $4 \times 4$ K Ultrascan 895 CCD (Gatan, USA) at a magnification of 30,000 (0.36 nm/pixel). Micrograph images were collected from the same sample area, but different exposure times were used to produce three micrograph datasets. The total dose of the sample exposed to the electron beam for 1 second was approximately $10^{5}$ electrons $/ \mathrm{nm}^{2}$ at a nominal underfocus of $2.5 \mu \mathrm{m}$. Micrographs were also taken after longer exposure times of 2 and 4 seconds.

\section{Comparative Analysis of Single Particle Image Processing}

Images of individual particles on the micrograph were selected interactively, windowed out, and imported into the SPIDER program suite (Health Research Inc., USA). To compare the electron densities produced after different exposure times, the same coordination profile obtained from the selection of particles in the 1 second exposure time micrograph was used to window out particles from the 2 and 4 seconds exposure time micrographs. A total of 200 well-separated particles were used in the alignment and classification, which were performed using the reference-free method described previously (Burgess et al., 2004). All subsequent single particle image processing was carried out using windowed datasets
A

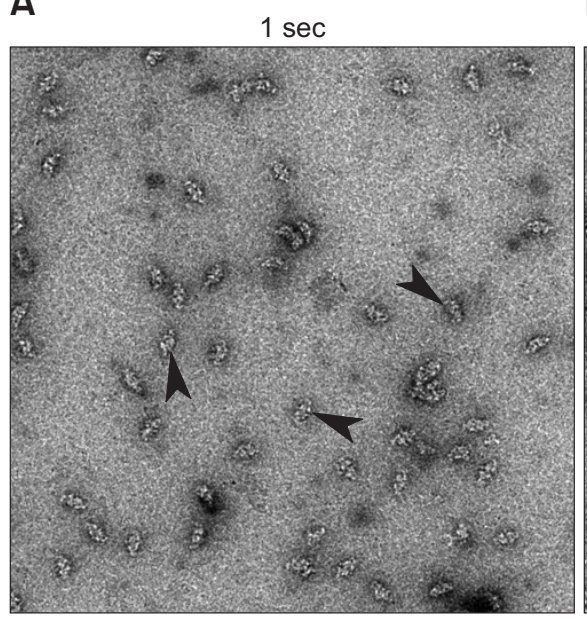

B

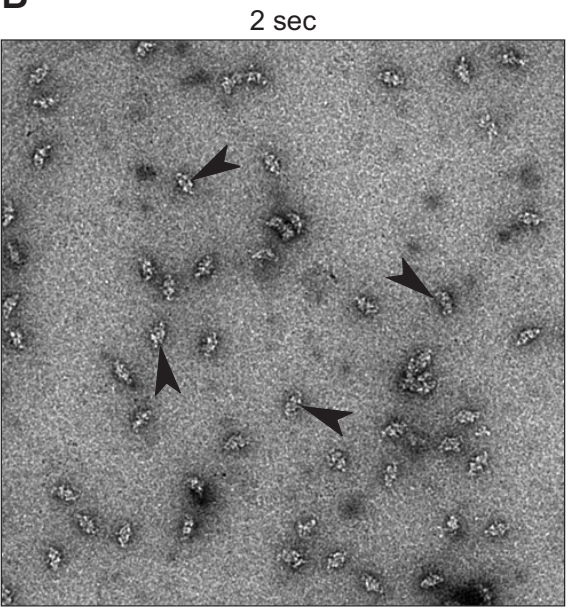

C

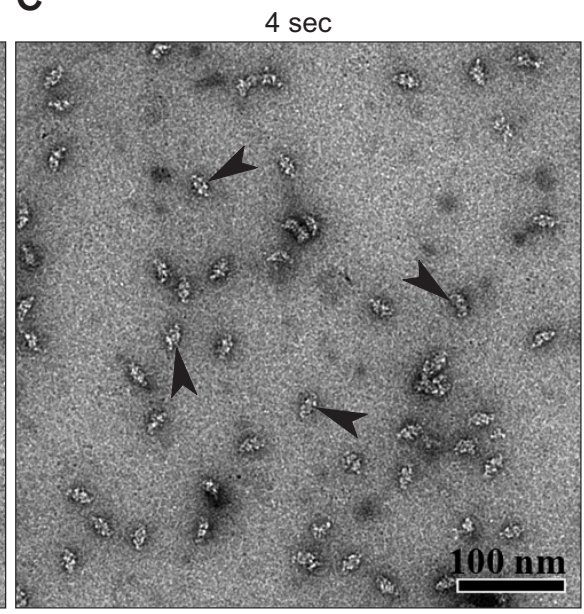

Fig. 1. Transmission electron microscopy images of the same sample site after three different electron beam exposure times. Negatively stained fields of protein samples exposed to the electron beam for 1 second (A), 2 seconds (B), 4 seconds (C). The arrowheads indicate representative molecules. Isolated thyroglobulin was used for the specimen preparation and 200 particles in each field were windowed for further analysis. Scale bar=100 nm. 
with the same alignment and classification parameters. A subtraction procedure was used to compare the structural differences between two $100 \times 100$ pixel averaged images processed from the same particles but obtained from electron densities resulting from different exposure times. The shorter exposure time image was subtracted from the longer exposure time image; the resulting subtracted image showed lighter shading in regions where the shorter exposure time image contained more density than the second image.

\section{RESULTS AND DISCUSSION}

\section{TEM Imaging of Negatively Stained Bio-Materials Exposed to an Electron Beam for Different Lengths of Time}

TEM images of negatively stained thyroglobulin (approximately $660 \mathrm{kDa}$ ) were collected after the same sample site was exposed to the after electron beam for 1,2, or 4 seconds (Fig. 1). In all three images, the molecules existed as appeared egg-shaped forms with lengths of $24 \sim 26 \mathrm{~nm}$ and widths of 11 12 nm (You et al., 2013). The appearances of the individual molecules after the different exposure times were indistinguishable (indicated by arrowheads in Fig. 1).

\section{Topographical Comparisons of the Averaging of Molecules}

Single particle image processing, digitized at a pixel size corresponding to $0.36 \mathrm{~nm}$, was used to compare the electron densities of the TEM images obtained using the three different exposure times. Individual molecules in the TEM images were selected interactively and windowed out into $100 \times 100$ pixels for processing, including alignment and classification. The

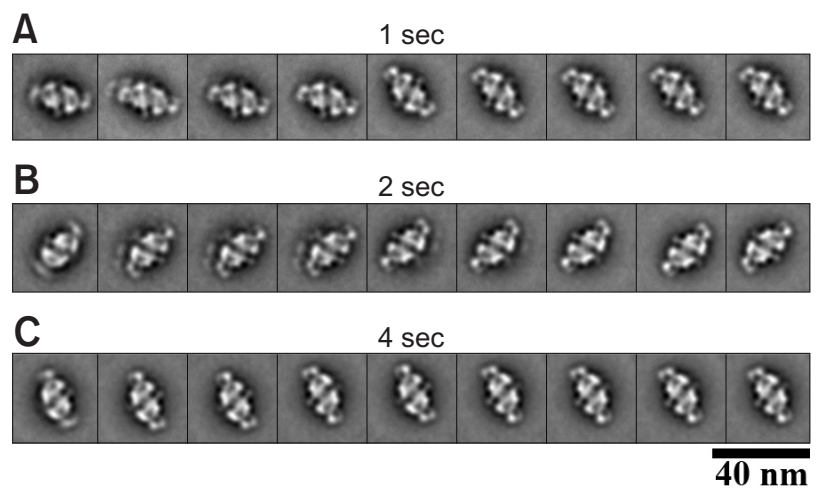

Fig. 2. Iterative alignments of transmission electron microscopy images after three different electron beam exposure times. Nine iterative alignments (left to right) of datasets after exposure for 1 second (A), 2 seconds (B), or 4 seconds (C). The alignments were performed with 200 particles in each dataset. Scale bar $=40 \mathrm{~nm}$.
A

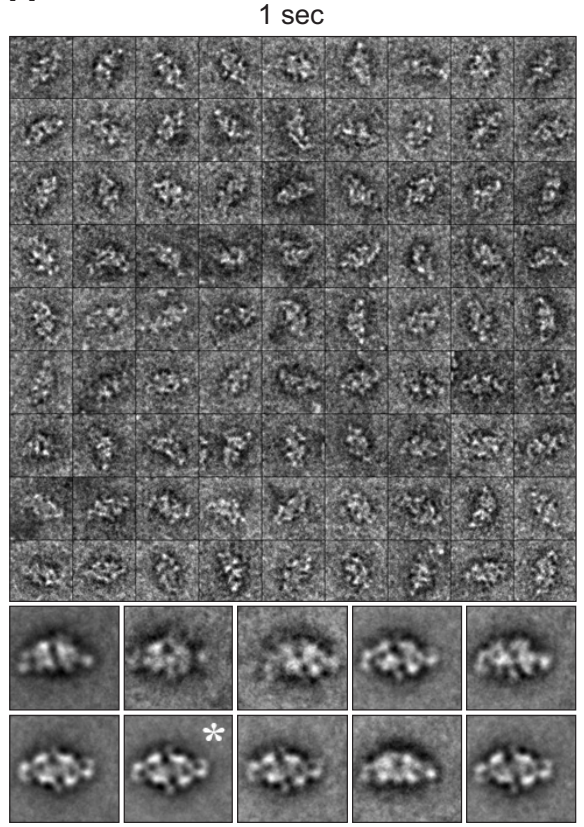

B

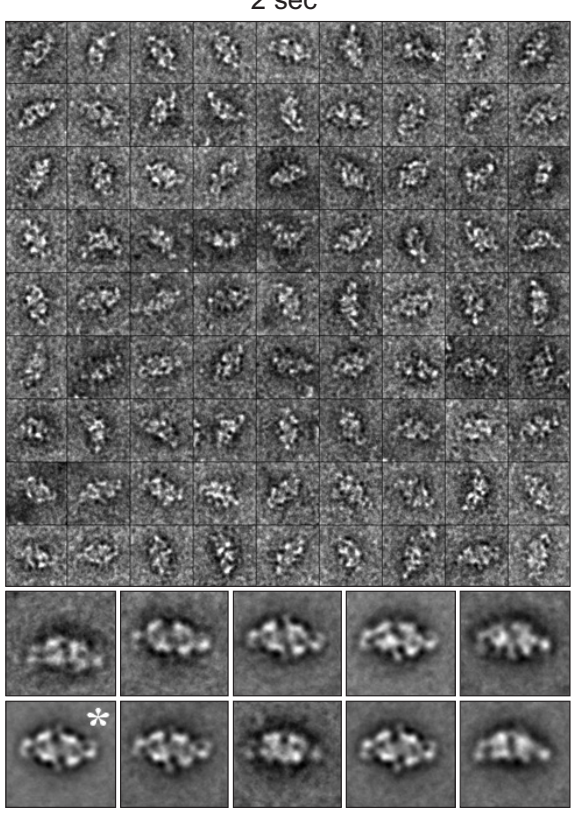

C

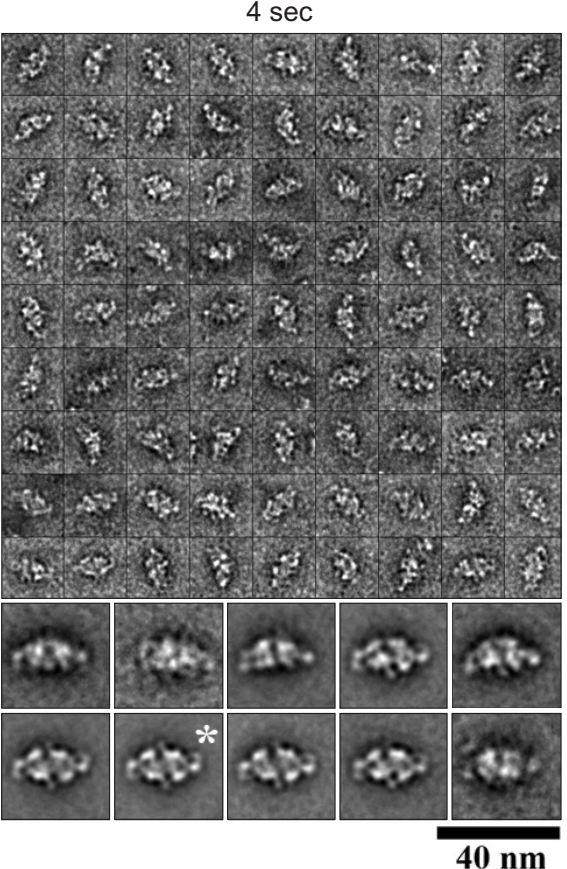

Fig. 3. (A-C) Windowed particles used in single particle analysis and their averaged images. Selected individual particles windowed from negatively stained fields (upper panels) and averaged images (bottom panels) from the three different exposure time datasets. The asterisks indicate the averaged image showing detailed densities that were selected for further comparative analyses. Each class average contained $15 \sim 25$ individual particles. Scale bar=40 nm. 
A
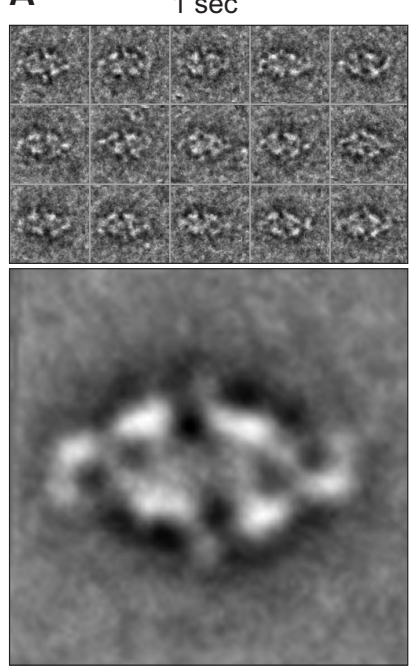

B

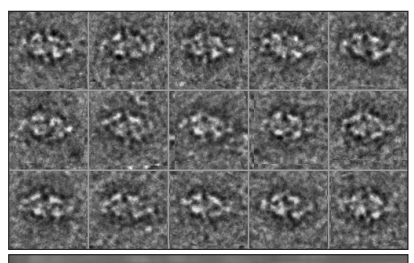

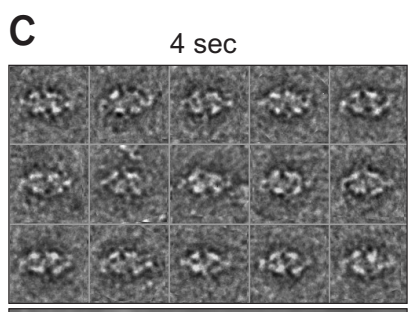

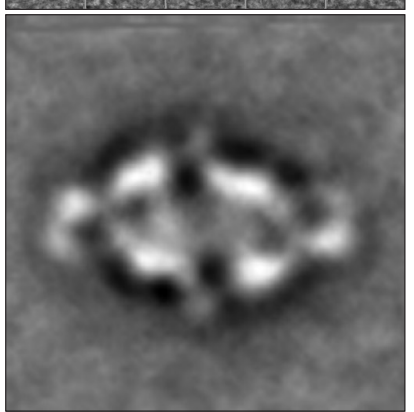

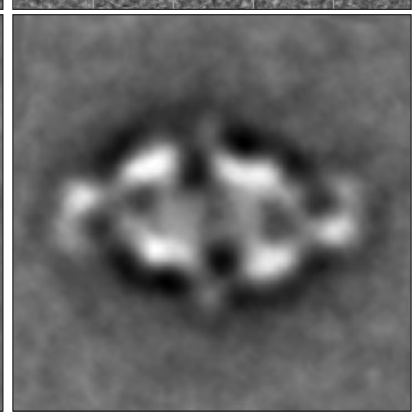

Fig. 4. Structural similarities and subtle differences between selected averaged images. Galleries of the constituent individual particles (upper panels) and enlarged averaged images (bottom panels) from the 1 second (A), 2 seconds (B), or 4 seconds (C) datasets (taken from the asterisk-marked averages shown in Fig. 3). To avoid objective comparisons of the densities of the three averaged images, only co-existing particles that contributed to all of the averages (15 particles in each dataset) were segregated and re-averaged. datasets were independently aligned with iterative alignments using the same parameters. The aligned images appeared very similar, but subtle differences between them were identified (Fig. 2). Fig. 3 shows windowed individual particles in each dataset and class averages processed from their alignments. A comparison of the raw images of the individual particles with the averaged images revealed a vast improvement in the level of detail after averaging. The averaged images from the 1 second exposure time dataset did not correspond well to those from the 2 and 4 seconds datasets (Fig. 3), indicating that the aligned particles provided different electron densities resulting in different class averaging details.

\section{Detection of Additional Electron Densities Induced by Longer Exposure Times}

To clarify and detect recognizable differences between the electron densities of the averaged images taken from the 1 , 2 , and 4 seconds datasets, the constituent images of selected averages showing structural details with similar oriented views (asterisk-marked averages in Fig. 3) were segregated from their original datasets. The same particle numbers and the same molecules were reassembled into the averages (Fig. 4), with the aim of avoiding subjectivity in determining the differences between the electron densities. Although the same molecules were used from each dataset, structural details between the averaged images were identified.

To reveal additional electron densities, averaged images of longer exposure time samples were subtracted from those of the shorter exposure time samples (Fig. 5). The resulting subtracted images showed lighter shading distributed within the averages (indicated by arrowheads in Fig. 5B), reflecting more electron densities in the images from the 4 seconds exposure time samples. This finding indicates that a longer exposure time (4 seconds) resulted in additional electron

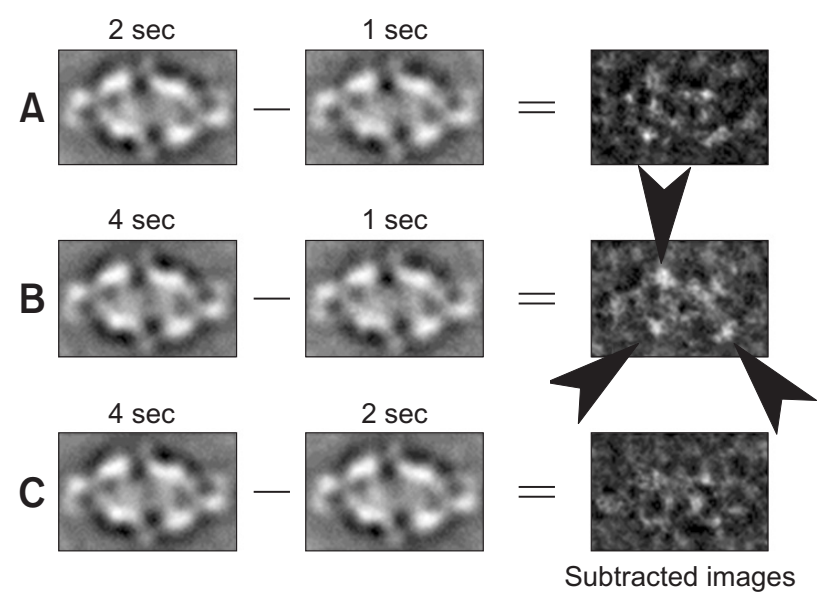

Fig. 5. (A-C) Comparative analysis of averaged images after subtraction. The averaged images of the longer exposure time samples were subtracted from those of the shorter exposure time samples. Note that subtraction process were required to define consistent structural differences between two images shown as white shaded areas in the subtracted images. Before applying the subtraction procedures, selected averaged images (taken from Fig. 4) were co-aligned and co-classified. The arrowheads indicate the positions of additional densities found in the subtracted images.

densities in the averaged images, possibly resulting in better alignment and class averaging.

Single bio-materials, such as protein molecules, have low molecular weights; hence, the information obtained from TEM imaging of each particle is limited. Previous studies (Henderson, 1995) using theoretical considerations demonstrated that unstained molecules larger than approximately $100 \mathrm{kDa}$ can be reconstructed to provide structural details. This molecular weight limit does not apply to negative staining TEM of proteins as well as cryo-EM (Kim et al., 2015); therefore, the use of correct instrumentation and optimized analytical parameters can maximize the quality of 
the structural densities obtained, potentially aiding structural determination of bio-materials at the molecular level.

\section{CONCLUSIONS}

The results presented here suggest that longer electron beam exposure times provide more electron densities of biomaterials analyzed by TEM imaging, ultimately resulting in optimal visualization of their detailed structural features. In general, generating raw images containing sufficient contrast and electron density is a fundamental requirement of morphological and structural analyses; thus, the evaluation procedures described here are a useful tool for maximizing the electron densities of TEM images for structural determinations of bio-materials at the molecular level.

\section{CONFLICT OF INTEREST}

No potential conflict of interest relevant to this article was reported.

\section{ACKNOWLEDGMENTS}

We thank Hyun Ju Lee for assisting with the TEM imaging.

\section{REFERENCES}

Anderson L J, Boyles J K, and Mahmood Hussain M (1989) A rapid method for staining large chylomicrons. J. Lipid Res. 30, 1819-1824.

Boekema E J, Folea M, and Kouril R (2009) Single particle electron microscopy. Photosynth Res. 102, 189-196.

Brenner S and Horne R W (1959) A negative staining method for high resolution electron microscopy of viruses. Biochim. Biophys. Acta 34, 103-110.

Burgess S A, Walker M L, Thirumurugan K, Trinick J, and Kinight P J (2004) Use of negative stain and single-particle image processing to explore dynamic properties of flexible macromolecules. J. Struct. Biol. 147, 247-258.

De Carlo S and Harris J R (2011) Negative staining and cryo-negative staining of macromolecules and viruses for TEM. Micron. 42, 117131.

Elliott P R, Irvine A F, Jung H S, Tozawa K, Pastok M W, Picone R, Badyal S K, Basran J, Rudland P S, Barraclough R, Lian L Y, Bagshaw C R, Kriajevska M, and Barsukov I L (2012) Asymmetric mode of Ca2+S100A4 interaction with nonmuscle myosin IIA generates nanomolar affinity required for filament remodeling. Structure 20, 654-666.

Henderson R (1995) The potential and limitations of neutrons, electrons and $\mathrm{x}$-rays for atomic resolution microscopy of unstained biological molecules. Q. Rev. Biophys. 28, 171-193.

Jaitly N, Brubaker M A, Rubinstein J L, and Lilien R H (2010) A bayesian method for $3 \mathrm{D}$ macromolecula $r$ structure inference using class average images from single particle electron microscopy. Bioinformatics 26, 2406-2415.

Jung $\mathrm{H} \mathrm{S}$, Billington $\mathrm{N}$, Thirumurugan $\mathrm{K}$, Salzameda $\mathrm{B}$, Cremo $\mathrm{C}$, Chalovich J M, Chantler P D, and Knight P J (2011) Role of the tail in the regulated state of myosin 2. J. Mol. Biol. 408, 863-878.

Jung H S, Komatsu S, Ikebe M, and Craig R (2008) Head-head and head- tail interaction: a general mechanism for switching off myosin II activity in cells. Mol. Biol. Cell 19, 3234-3242.

Kim J, Wu S, Tomasiak T, Mergel C, Winter M B, Stiller S B, RoblesColmanares Y, Stroud R M, Tampé R, Craik C S, and Cheng Y (2015) Subnanometer resolution cryo-EM structure of a nucleotide free heterodimeric ABC exporter. Nature 517, 396-400.

Lee S, Jia B, Liu J, Pham B P, Kwak J M, Xuan Y H, and Cheong G W (2015). A 1-cys peroxiredoxin from a thermophilic archaeon moonlights as a molecular chaperone to protect protein and DNA against stressinduced damage. PLOS ONE 10, e0125325.

Massover W H (2008) Negative staining produces images of proteins by which contrast mechanisms? Microsc. Microanal. 14, 672-673.

Monroe J H and Brandt P M (1970) Rapid semiquantitative method for screening large numbers of virus samples by negative staining electron microscopy. Appl. Microbiol. 20, 259-262.

Murata K, Liu X, Danev R, Jakana J, Khant H, Schmid M F, Nagayama K, and Chiu W (2010) Zernike phase contrast cryo-EM for biological structure determination. Microsc. Microanal. 16, 544-545.

Nagayama K and Danev R (2004) Phase contrast electron microscopy: development of thin-film phase plates and biological applications. Phil. Trans. R. Soc. B 363, 2153-2162.

Umeki N, Jung H S, Watanabe S, Sakai T, Li X, Ikebe R, Craig R, and Ikebe $M(2009)$ The tail binds to the head-neck domain, inhibiting ATPase activity of myosin VIIA. Proc. Natl. Acad. Sci. USA 106, 8483-8488.

You D J, Jhon G J, and Jung H S (2013) Molecular assembly of thyroglobulin induced by in vitro nitric oxide treatments: implication its role in thyroid cells. Protein J. 32, 619-625.

Zhang L, Tong H, Garewal M, and Ren G (2013) Optimized negativestaining electron microscopy for lipoprotein studies. Biochim. Biophys. Acta 1830, 2150-2159. 